\title{
Tecnologias Educacionais sobre Cuidados Paliativos no Instagram e no Youtube
}

\author{
Educational Technology about Palliative Care on Instagram and Youtube \\ Tecnologías Educativas sobre Cuidados Paliativos en Instagram y Youtube
}

Recebido: 26/05/2021 | Revisado: 04/06/2021 | Aceito: 04/06/2021 | Publicado: 18/06/2021

\author{
Nataniele Kmentt da Silva \\ ORCID: https://orcid.org/0000-0001-9798-6547 \\ Universidade Federal de Pelotas, Brasil \\ E-mail: nat.kmentt.s@gmail.com \\ Júlia Brombila Blumentritt \\ ORCID: https://orcid.org/0000-0001-8455-5596 \\ Universidade Federal de Pelotas, Brasil \\ E-mail: juliabrombila@hotmail.com \\ Franciele Roberta Cordeiro \\ ORCID: https://orcid.org/0000-0001-6194-5057 \\ Universidade Federal de Pelotas, Brasil \\ E-mail: franciele.cordeiro@ufpel.edu.br
}

\begin{abstract}
Resumo
Mídias sociais são uma forma de realizar ações de educação em saúde. Assim, este artigo tem por objetivo relatar o uso de tecnologias educacionais, especificamente vídeos e folhetos, sobre cuidados paliativos no Instagram e no YouTube. Trata-se de um relato de experiência, realizado entre junho de 2020 e fevereiro de 2021, por docentes e discentes vinculados a um projeto de extensão. O Instagram e o YouTube foram o cenário da experiência. Como resultados, foram construídos 24 folhetos e vídeos educativos sobre cuidados paliativos. Os temas versaram sobre aspectos clínicos, sociais, econômicos e o luto. Tais materiais foram publicados em perfis do projeto. Em relação aos usuários, a maioria era do sexo feminino, na faixa etária entre 25-34 anos. O acesso aos materiais ocorreu, majoritariamente, através de dispositivos móveis. No Instagram, o tema do vídeo mais visualizado foi a importância do sono no final da vida. Quanto aos folhetos, os que tiveram melhores impressões foram aqueles sobre alívio nãofarmacológico da dor, controle de náuseas e vômitos e suspensão de terapias renais substitutivas no final da vida. No YouTube, o vídeo com maior alcance foi sobre diretivas antecipadas de vontade. Conclui-se que o Instagram e o YouTube são mídias sociais com potencial de disseminação de tecnologias educacionais, por serem de fácil acesso, podendo ser utilizados pela enfermagem para o desenvolvimento de ações educativas.
\end{abstract}

Palavras-chave: Tecnologia educacional; Cuidados paliativos; Mídias sociais.

\begin{abstract}
Social media is a way to carry out health education actions. to report the use of educational technologies, specifically videos, and brochures, on palliative care on Instagram and YouTube. Thus, this article aims to report the use of innovative technology, carried out between June 2020 and February 2021, by professors and students linked to an extension project. Instagram and YouTube were the settings for the experience. We constructed 24 leaflets and educational videos on palliative care. The themes dealt with clinical, social, economic, and mourning aspects. We published these materials in project profiles. About users, the majority were female, in the age group between 25-34 years. Access to the materials was predominantly through mobile devices. On Instagram, the most viewed theme was the importance of sleep at the end of life and control of dyspnea. As for the leaflets, the ones with the best impressions were those on non-pharmacological pain relief, management of nausea and vomiting, and withdrawal of renal replacement therapies at the end of life. On YouTube, a video with greater reach was about advanced directives. We concluded that Instagram and YouTube are social media with the potential to disseminate educational technologies because they are easily accessible and can be employed by nursing to develop educational actions.
\end{abstract}

Keywords: Educational technology; Palliative care; Social media.

\section{Resumen}

Las redes sociales son una forma de realizar acciones de educación para la salud. Así, este artículo tiene como objetivo reportar la experiencia con el uso de tecnologías educativas, específicamente videos y folletos, sobre cuidados paliativos en Instagram y YouTube. Se trata de un relato de experiencia, realizado entre junio de 2020 y febrero de 2021, por docentes y estudiantes vinculados a un proyecto de extensión. Instagram y YouTube fueron el escenario de la experiencia. Se construyeron 24 folletos y videos educativos sobre cuidados paliativos. Los temas contemplaron aspectos clínicos, sociales, económicos y el luto. Los materiales fueron publicados en los perfiles del proyecto. Mujeres, en la franja de edad entre 25-34 años, eran mayoría de los usuarios. El acceso se produjo 
principalmente a través de dispositivos móviles. En Instagram, el tema del video más visto más visualizado fue sobre la importancia del sueño al final de la vida. En cuanto a los folletos, aquellos sobre alivio no farmacológico del dolor y control de náuseas, vómitos tuvieron mejores impresiones y suspensión de las terapias de reemplazo renal al final de la vida. En YouTube, el video con mayor alcance fue sobre directivas anticipadas de voluntad. Se concluye que Instagram y YouTube son redes sociales con potencial de diseminación de tecnologías educativas, por la facilidad de acceso, pudiendo ser utilizados por la enfermería para el desarrollo de actividades educativas.

Palabras clave: Tecnología educacional; Cuidados paliativos; Medios de Comunicación Sociales.

\section{Introdução}

Mídias sociais são plataformas que permitem a expressão e o compartilhamento de sentimentos e opiniões, além do engajamento em torno de causas e problemas comuns. Com a internet, elas passaram a mediar interações em que cada um se torna ator ativo da comunicação e da relação com os demais na busca por produtos, pessoas, serviços e informações (Li et al., 2021).

Considerando o alcance das mídias sociais na vida cotidiana, profissionais e pesquisadores da área da saúde têm utilizado essas plataformas para educar pessoas sobre doenças específicas, como por exemplo, a Coronavirus Disease 2019 (Covid-19) - combatendo informações falsas (Souza et al., 2020), orientar sobre cuidados paliativos domiciliares (OllerArlandis \& Oller-Arlandis, 2017) ou dar visibilidade à identidade profissional (Lima et al., 2020). Em se tratando de educação em saúde, elas podem ser utilizadas para difundir tecnologias educacionais, pois afetam um maior número de pessoas, modificando comportamentos.

Tecnologias educacionais, no contexto da saúde, são caracterizadas como um conjunto de conhecimentos passível de ser implementado por meio de produtos ou processos para facilitar o ensino-aprendizagem (Nietsche et al., 2005). Elas podem ser acionadas visando à promoção ou à recuperação da saúde, à prevenção de doenças e à paliação, devendo considerar a linguagem e o uso de ferramentas compreensíveis e adaptáveis ao público para o qual elas se direcionam (Maniva et al., 2018).

Nesse sentido, torna-se relevante, ao abordar educação em saúde, recorrer às tecnologias educacionais que sejam de fácil acesso. Vídeos, cartilhas, infográficos e folhetos têm apresentado força de recomendação para uso na prática clínica e na formação em saúde (Westphal Hahn \& Cordeiro, 2021). As mídias sociais permitem sua maior circulação, já que são acessadas, majoritariamente, de dispositivos móveis, como por exemplo, smartphones (Lee et al., 2015).

Frente ao exposto, o presente artigo tem como objetivo relatar o uso de tecnologias educacionais, especificamente vídeos e folhetos, sobre cuidados paliativos no Instagram e no YouTube.

\section{Metodologia}

Estudo de abordagem qualitativa, descritivo, do tipo relato de experiência, desenvolvido entre junho de 2020 e fevereiro de 2021, nas mídias sociais Instagram e YouTube. Os participantes envolvidos foram discentes de graduação e pósgraduação em enfermagem, docentes e profissionais de saúde vinculados ao projeto de extensão "A consulta de enfermagem como instrumento de cuidado às pessoas com doenças que ameaçam a vida e suas famílias". Além disso, foram alvo da experiência os usuários e as usuárias dos perfis no Instagram e no YouTube para divulgação dos vídeos e folhetos sobre cuidados paliativos.

O Instagram é uma mídia social fundada em 2010, que pertence à empresa Facebook. Tem por objetivo conectar pessoas, tanto aquelas com contato próximo como as denominadas "comuns", com celebridades e instituições (Lee et al., 2015). Diferentemente de outras mídias sociais, o foco do Instagram é o compartilhamento de imagens e vídeos, conforme o apresentado pela própria empresa, para permitir a expressão de si dentro de uma comunidade (Instagram do Facebook, 2021). Nele são encontrados perfis relacionados às áreas da saúde, educação, jornalismo, moda, comportamento, além de empresas ou pessoas autônomas que o utilizam como uma ferramenta comercial. Dentre as opções de interação e avaliação do impacto das 
publicações estão as opções "gostei", comentar, salvar, compartilhar/encaminhar, visualizações, impressões e descobertas. As impressões estão relacionadas ao número de vezes que uma postagem é exibida e as descobertas ao número de contas atingidas, independentemente de estar seguindo, ou não, determinado perfil.

O YouTube é uma plataforma de compartilhamento de vídeos, criada em 2005, pertencente à empresa Google. Tem por finalidade a expressão de opinião, a promoção do diálogo e a divulgação de diferentes informações através do recurso audiovisual. Também visa à formação de comunidades que debatam temas de interesse comum (Sobre o YouTube - YouTube, [s.d.]). É crescente o uso dessa plataforma por instituições, pacientes e profissionais de saúde para compartilhar orientações sobre cuidados, experiências e também como fonte de orientação (Liu et al., 2019; Mitchell et al., 2017). No YouTube, o número de visualizações é o principal meio de avaliar o impacto e a influência de cada publicação (Mitchell et al., 2017).

Até janeiro de 2021, estimava-se que aproximadamente 3,6 bilhões de pessoas no mundo possuíam um perfil em, pelo menos, uma mídia social. O Instagram era a $5^{\circ}$ e o YouTube era a $2^{\circ}$ com mais usuários ativos (Most Used Social Media 2020, 2021). No Brasil, cerca de 141,5 milhões de pessoas fazem uso de alguma mídia social. O Instagram, até 2019, contava com aproximadamente 69 milhões de usuários e o YouTube com 147 milhões (Statista, 2021).

Assim, o objetivo da experiência foi divulgar os cuidados paliativos para a população, priorizando os aspectos clínicos que envolvem o acompanhamento junto às pessoas com doenças que ameaçam a vida, os princípios e conceitos inerentes a essa abordagem, bem como os aspectos éticos e sociais que envolvem o final da vida.

Como os resultados apresentados neste artigo são oriundos de dados de domínio público, dispensa-se a aprovação do Comitê de Ética em Pesquisa, considerando o disposto nas Resoluções 466/2012 e 510/2016.

\section{Resultados e Discussão}

As tecnologias educacionais desenvolvidas foram vídeos e folhetos. Para a construção de ambos foi elaborado semanalmente um roteiro prévio, sustentado em literatura pertinente ao tema, ou seja, manuais e diretrizes de cuidados paliativos nacionais e internacionais, portarias ministeriais, consensos e artigos extraídos de periódicos em bases de dados. Cada roteiro foi revisado e validado por profissionais vinculados a uma equipe de consultoria em cuidados paliativos ou docentes dos cursos de Graduação em Enfermagem ou Medicina. Utilizou-se linguagem o mais próximo da coloquial, a fim de promover o entendimento dos conteúdos tanto por profissionais de saúde quanto pelo público leigo.

Os folhetos foram criados na plataforma Canva, em sua versão profissional. Todos apresentavam o mesmo leiaute, composto de duas colunas, divididas em quadros menores, de fundo rosa claro. A fonte utilizada em todos os materiais foi Open Sans Light, na cor azul. Na coluna inicial, introduziu-se o assunto, com uma breve definição do tema, sendo utilizada, em sua maioria, a estratégia de perguntas e respostas. Além de textos, cada quadro continha imagens que representavam o assunto nele abordado. Na parte superior anunciava-se o título geral do folheto seguido do subtítulo que representava a temática abordada. Na parte inferior descrevia-se as referências utilizadas e a autoria, com o nome do projeto de extensão. Na Figura 1, apresenta-se os folhetos com maior número de impressões a fim de exemplificar o modelo utilizado. 
Figura 1 - Folhetos com maior número de impressões no Instagram.

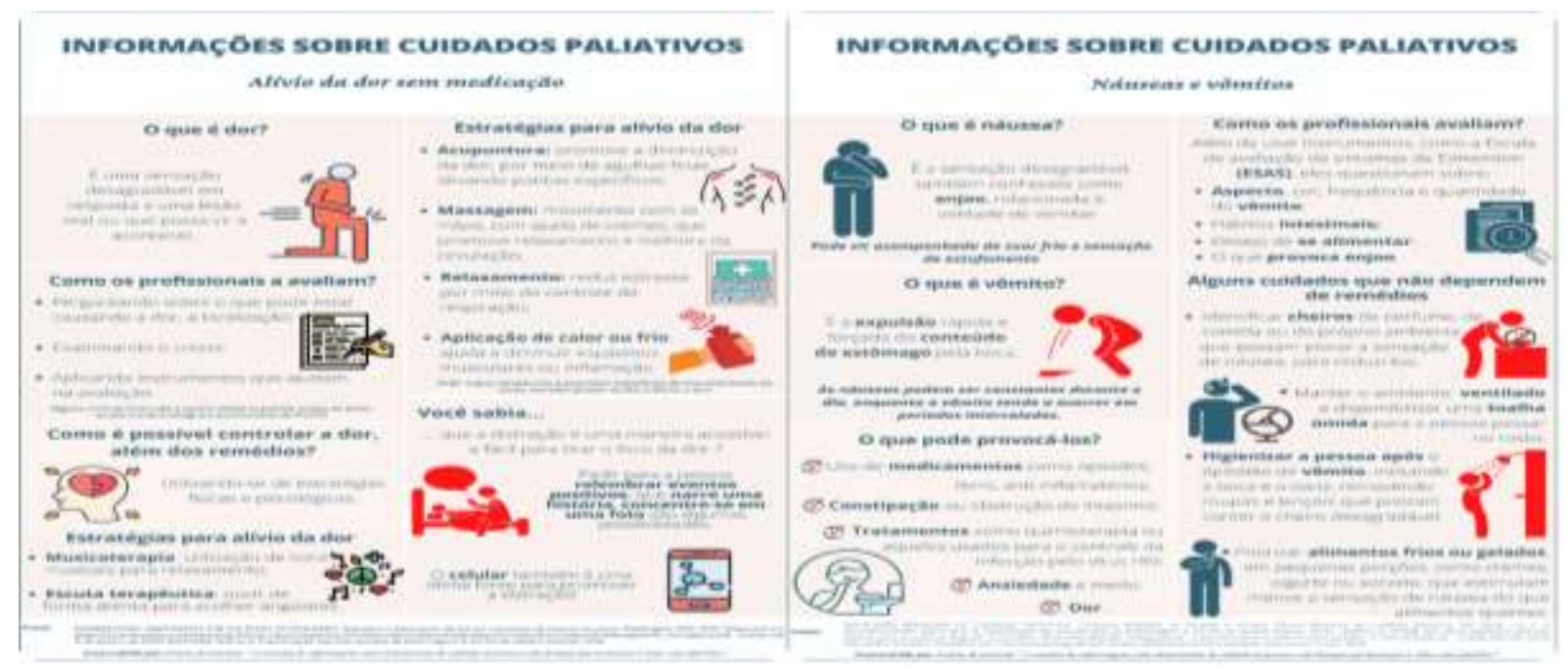

Fonte: Dados da pesquisa (2021).

Os vídeos foram criados, tendo como base a plataforma Canva, entretanto, também foram editados no programa Inshot, em sua versão gratuita. Assim como os folhetos, os vídeos possuíam identificação padronizada, a qual continha um quadro na capa e dentro desta o título da temática abordada. Para cada tema foi utilizada uma paleta de cores específica. No canto superior direito do quadro com o título inseria-se a imagem de uma borboleta, a qual foi utilizada em todos os vídeos por ser considerada o símbolo dos cuidados paliativos. Na cena final de cada vídeo foi mencionado o financiamento para realização dos materiais, a instituição à qual estava vinculada a produção, bem como o endereço das redes sociais do projeto. Os vídeos tiveram duração que variou entre 5 minutos e 53 segundos e 19 minutos e 35 segundos.

Os materiais foram divulgados e continuam disponíveis nos perfis do projeto de extensão, criados no YouTube (GEAFI - Cuidados Paliativos UFPEL) e no Instagram (@geafi), sendo que nesta última o perfil criado foi do tipo profissional. No quadro 1 estão apresentados os 24 vídeos e 24 folhetos construídos. 
Quadro 1 - Vídeos e folhetos construídos.

\begin{tabular}{|c|c|c|c|}
\hline Vídeo & Folheto & Tema & Data da publicação \\
\hline V1 & F1 & Cuidados paliativos: definições & $17 / 06 / 2020$ \\
\hline $\mathbf{V} 2$ & F2 & Identificação da necessidade de cuidados paliativos & $24 / 06 / 2020$ \\
\hline $\mathbf{V 3}$ & F3 & Serviços de cuidados paliativos & $01 / 07 / 2020$ \\
\hline V4 & F4 & Diretivas antecipadas de vontade & $08 / 07 / 2020$ \\
\hline V5 & F5 & Transição do cuidado no final da vida & $15 / 07 / 2020$ \\
\hline V6 & F6 & Importância do sono no final da vida & $22 / 07 / 2020$ \\
\hline V7 & F7 & Últimas horas de vida & $29 / 07 / 2020$ \\
\hline V8 & F8 & Cuidado à família & $05 / 08 / 2020$ \\
\hline V9 & F9 & Controle da dispneia & $12 / 08 / 2020$ \\
\hline V10 & F10 & Controle da dor com medidas não-farmacológicas & $19 / 08 / 2020$ \\
\hline V11 & F11 & Controle da dor com medidas farmacológicas & $09 / 09 / 2020$ \\
\hline V12 & F12 & Hipodermóclise & $16 / 09 / 2020$ \\
\hline V13 & F13 & Alimentação e nutrição em cuidados paliativos & $23 / 09 / 2020$ \\
\hline V14 & F14 & Hidratação no final da vida & $30 / 09 / 2020$ \\
\hline V15 & F15 & Cuidados com a pele no final da vida & $07 / 10 / 2020$ \\
\hline V16 & F16 & Controle da constipação & $14 / 10 / 2020$ \\
\hline V17 & F17 & Controle de náuseas e vômitos & $21 / 10 / 2020$ \\
\hline V18 & F18 & Delirium e ansiedade & $28 / 10 / 2020$ \\
\hline V19 & F19 & Suspensão de ventilação mecânica no final da vida & $04 / 11 / 2020$ \\
\hline $\mathbf{V 2 0}$ & F20 & $\begin{array}{l}\text { Suspensão de terapias renais substitutivas no final da } \\
\text { vida }\end{array}$ & $11 / 11 / 2020$ \\
\hline $\mathbf{V} 21$ & F21 & $\begin{array}{l}\text { Transformações do corpo no final da vida e } \\
\text { dignidade humana }\end{array}$ & $18 / 11 / 2020$ \\
\hline $\mathbf{V 2 2}$ & F22 & $\begin{array}{l}\text { Planejamento social e econômico em cuidados } \\
\text { paliativos }\end{array}$ & $25 / 11 / 2020$ \\
\hline $\mathbf{V} 23$ & F23 & Morte encefálica & $02 / 12 / 2020$ \\
\hline V24 & F24 & Luto & $09 / 12 / 2020$ \\
\hline
\end{tabular}

Fonte: Dados da pesquisa (2021). 
Em relação aos usuários que acompanham as redes sociais do projeto, no Instagram pode-se recuperar dados referentes à localização, à idade e ao sexo. No YouTube, além desses, também foram analisados: origem do tráfego, os dispositivos utilizados para o acesso aos vídeos, a duração média de visualização dos vídeos e o status de inscrição dos espectadores.

No Instagram, o perfil possui 501 seguidores. No que concerne à localização, 98,7\% são do Brasil, 0,9\% da Argentina, 0,2\% de Portugal e 0,2\% da Espanha, sendo que no Brasil, o público que mais acompanha o perfil é das cidades de Pelotas (46,6\%), Porto Alegre (4,7\%), São Paulo (3,0\%), Rio Grande (2,1\%) e Santa Maria (1,9\%). Quanto à idade do público, constata-se que os seguidores são predominantemente da faixa etária 25-34 (37,8\%) e de 18-24 (35,6\%) anos, seguido daqueles entre 35-44 (18,2\%), 45-54 (6,0\%) e 55-64 (2,0\%) anos. Em relação ao sexo, 86,3\% são mulheres e 13,7\% são homens.

O YouTube conta com 122 inscritos. Em relação à localização, 52,5\% do público é do Brasil. Quanto ao sexo, observou-se predominância do feminino (100\%). Em relação à idade, prevaleceu a faixa etária de 25-34 anos (53,3\%), seguida de 18-24 (46,7\%). No que concerne a origem do tráfego, ou seja, por onde foram acessados os vídeos, constatou-se predominância de: pesquisas do YouTube - realizadas diretamente pela plataforma através de palavras-chaves - $(53,7 \%)$, vídeos sugeridos dentro da própria plataforma $(18,2 \%)$ e origem externa - acesso a partir de websites ou aplicativos que incluem o link do vídeo - $(9,1 \%)$. No que tange aos dispositivos utilizados para visualização dos vídeos estão: dispositivos móveis $(66,9 \%)$, computador $(28,1 \%)$, tablet $(3,3 \%)$ e televisão $(1,7 \%)$. Quanto à duração média de visualização, os três mais vistos foram: V3, V4 e V7, com 10 minutos e 55 segundos, 7 minutos e 59 segundos e 5 minutos e 3 segundos, respectivamente. E no que se refere ao status de inscrição, constatou-se que $95 \%$ do público que assistiu aos vídeos não era inscrito no canal e apenas $5 \%$, inscrito.

Segundo análise sobre o perfil de mídias sociais no Brasil, 31\% do total de usuários do Instagram têm entre 25 e 34 anos e a maioria são mulheres (Statista, 2021). Tal dado converge com os obtidos neste relato, pois observou-se que em relação à idade e ao sexo dos usuários do Instagram e do YouTube, 37,8\% no Instagram e 53,3\% no YouTube está na faixa etária de 25-34 anos e 86,3\% no Instagram e 100\% no YouTube são do sexo feminino. Além disso, a plataforma YouTube possui uma taxa de 800 milhões de usuários/mês, sendo que 1/5 dos acessos acontece através de dispositivos móveis (Centers for Disease Control and Prevention, 2012), outro dado convergente aos achados deste relato.

A Figura 2 representa o total de visualizações dos vídeos no Instagram e no YouTube a partir do início dos registros, ou seja, um mês após a publicação dos materiais. 
Figura 2 - Visualizações dos vídeos no Instagram e no YouTube.

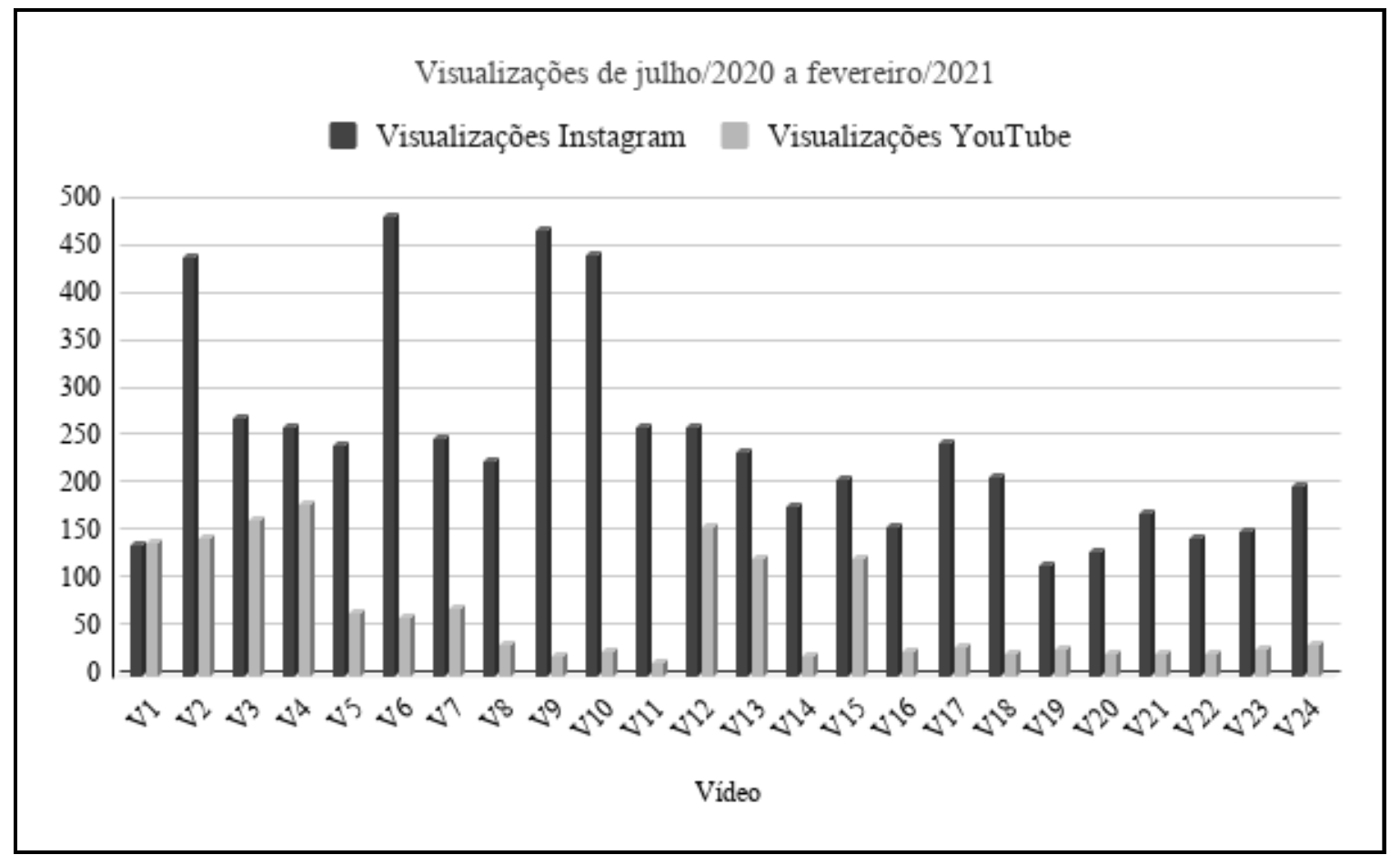

Fonte: Da Silva, Blumentritt e Cordeiro (2021).

Verifica-se que no Instagram, os vídeos V6, V9 e V10 tiveram 483, 469 e 443 visualizações, respectivamente, e no YouTube, os vídeos V4, V3 e V12 com 180, 156 e 143 visualizações, respectivamente, ao longo do período. Quanto ao número de visualizações dos vídeos, notou-se superioridade do Instagram em relação ao YouTube.

Acredita-se que essa variação ocorreu devido ao Instagram ser uma mídia social com grande apelo por reunir assuntos de interesse geral em único local, possuindo acesso médio de quatro vezes ao dia (Lee et al., 2015). Além disso, o Instagram conta com uma ferramenta de compartilhamento instantâneo de qualquer publicação para o stories pessoal do usuário, ou seja, a publicação pode ficar disponível por até 24 horas no perfil de quem a compartilhou, o que permite maior divulgação e visibilidade dos materiais postados.

Em relação ao vídeo mais visualizado no Instagram, a importância do sono foi o tema abordado. O sono, no final da vida e em cuidados paliativos, é responsável por manter as funções fisiológicas e a qualidade de vida. Dessa forma, o sono e o repouso devem ser constantemente avaliados e integrados no cuidado desses pacientes, pois a alteração do padrão sono-vigília interfere diretamente na qualidade de vida e conforto (Santos et al., 2016).

Durante a hospitalização, é possível haver alterações nos padrões de sono devido ao contexto hospitalar e à situação clínica dos pacientes. Dentre os estressores, estão: ruídos, iluminação, temperatura, conforto do leito, problemas psicológicos, dor, fadiga e interrupções do sono noturno para o recebimento de cuidados. Assim, considerando tais estressores, deve haver planejamento de intervenções, especialmente aquelas voltadas ao controle do ambiente, que minimizem interrupções e contribuam com a higiene do sono (Costa \& Ceolim, 2013).

Quanto ao vídeo com maior alcance no YouTube, o tema era sobre Diretivas Antecipadas de Vontade (DAV). As DAV são manifestações expressas pelos pacientes por escrito sobre tratamentos e cuidados de saúde que desejam ou não receber. São utilizadas para expressar vontades quando esses são acometidos por doenças graves e avançadas e já não conseguem mais comunicar sobre aquilo que desejam, permitindo que a equipe de saúde e a família conheçam e respeitem suas vontades (Dadalto, 2016; Dadalto et al., 2013). Podem ser de dois tipos: testamento vital, documento no qual o paciente tem o direito de aceitar ou recusar cuidados de saúde e procedimentos médicos que deseja ou não receber ou mandato duradouro, 
documento no qual o paciente nomeia alguém de confiança para tomar decisões por ele no momento em que não for mais capaz de decidir por si (Dadalto et al., 2013).

O Quadro 2 apresenta as impressões e as descobertas dos folhetos publicados no Instagram.

Quadro 2 - Impressões e descobertas dos folhetos no Instagram.

\begin{tabular}{|c|c|c|c|}
\hline \multirow{3}{*}{$\begin{array}{l}\text { Plataforma } \\
\text { Material }\end{array}$} & \multicolumn{3}{|l|}{ Instagram } \\
\hline & \multicolumn{2}{|l|}{ Impressões } & \multirow{2}{*}{\begin{tabular}{|l} 
Descobertas \\
$07 / 2020-02 / 2021$
\end{tabular}} \\
\hline & $\begin{array}{l}\text { Mês } 1 \\
(07 / 2020)\end{array}$ & $\begin{array}{l}\text { Mês } 2 \\
(08 / 2020)\end{array}$ & \\
\hline F1 & 227 & 238 & 184 \\
\hline F2 & 228 & 231 & 181 \\
\hline F3 & 240 & 247 & 185 \\
\hline F4 & 210 & 220 & 167 \\
\hline F5 & 215 & 224 & 184 \\
\hline F6 & 225 & 239 & 183 \\
\hline F7 & 220 & 231 & 187 \\
\hline F8 & 216 & 223 & 174 \\
\hline F9 & 244 & 249 & 202 \\
\hline F10 & 278 & 288 & 221 \\
\hline F11 & 243 & 248 & 202 \\
\hline F12 & 211 & 218 & 176 \\
\hline F13 & 208 & 222 & 164 \\
\hline F14 & 241 & 250 & 198 \\
\hline F15 & 236 & 246 & 198 \\
\hline F16 & 245 & 254 & 204 \\
\hline F17 & 272 & 274 & 211 \\
\hline F18 & 232 & 236 & 191 \\
\hline F19 & 209 & 213 & 165 \\
\hline F20 & 252 & 254 & 211 \\
\hline F21 & 189 & 190 & 150 \\
\hline F22 & 179 & 182 & 152 \\
\hline F23 & 236 & 238 & 207 \\
\hline F24 & 187 & 190 & 163 \\
\hline
\end{tabular}


No que diz respeito aos folhetos, F10, F17 e F20 tiveram mais impressões e descobertas. Os temas abordados nesses folhetos foram controle da dor com medidas não-farmacológicas, controle de náuseas e vômitos e suspensão de terapias renais substitutivas no final da vida.

A dor é um dos sintomas mais comuns do final da vida que interfere nas atividades de vida diária. Considera-se que é uma experiência única para cada pessoa e pode ser desencadeada por fatores biológicos, emocionais, psicológicos e sociais. Pode-se subdividir em leve, moderada e grave, segundo a intensidade, e em aguda e crônica, de acordo com o tempo de duração, sendo a primeira caracterizada por aparecimento repentino, relacionada a um evento isolado e a segunda pode ser intensa e contínua, devido a um distúrbio crônico. Para cada paciente é preciso avaliar o tipo de dor possibilitando uma conduta adequada, para auxiliar nessa avaliação existem escalas que podem ser utilizadas (IASP, 2010; IASP Terminology IASP, [s.d.]; Oliveira et al., 2019).

Após classificar o tipo de dor, algumas medidas não-farmacológicas podem ser implementadas, tanto com, quanto sem medicamentos. Dentre elas, cita-se: acupuntura, massagens, toque terapêutico, musicoterapia, atividades físicas, técnicas de relaxamento, distração, escuta terapêutica, aplicação de calor ou frio, promoção de repouso e sono e controle do ambiente. Todas essas abordagens, permitem alívio do desconforto causado pela dor, além de auxiliar no controle de ansiedade e depressão, dentre outros sintomas desagradáveis no final da vida, inclusive, náuseas e vômitos (IASP, 2010; Lewis et al., 2018).

As náuseas e os vômitos podem trazer desconforto para os pacientes que experienciam o final da vida, afetando diretamente sua qualidade de vida. Esses sintomas podem estar relacionados ao uso de alguns medicamentos, constipação ou obstrução intestinal, tratamentos quimioterápicos, distúrbios renais, dor, ansiedade e depressão. Pode-se identificá-los a partir do relato do paciente ou de seus familiares através de investigação sobre padrões alimentares, aversão a odores, histórico de saúde e de doenças, padrões de eliminação intestinal, vesical e estomacal, observando as características de cada uma delas. É importante proceder à avaliação nutricional, condições de desidratação, alterações fisiológicas na mucosa oral, além do estado emocional, financeiro e social. Para mensuração, existe a Escala de Avaliação de Sintomas de Edmonton (ESAS), a qual permite avaliação das náuseas contribuindo para o diagnóstico e conduta adequados (Associação Brasileira de Cuidados Paliativos, 2013; Monteiro et al., 2013; Palliaguide, [s.d.]).

Para efetuar o controle desses sintomas, após identificados, pode-se proceder a abordagem farmacológica ou nãofarmacológica, dependendo da necessidade do paciente. Como medida farmacológica, cita-se a metoclopramida, haloperidol ou ondansetrona, como os mais utilizados os quais diferenciam-se por seu mecanismo de ação. Já, no que tange a conduta nãofarmacológica, pode-se mencionar o uso de gengibre, estimulação elétrica transcutânea (TENS), acupuntura e relaxamento. É importante identificar os fatores desencadeantes desses sintomas para que sejam minimizados, encorajar o paciente a relatar quando sentir-se desconfortável, oferecer líquidos frios, caso a pessoa tenha condições de ingestão, orientar a pessoa a manterse com a cabeça mais elevada quanto seja possível, para que se reduza a sensação de desconforto, além de manter cuidados de higiene após os episódios de vômitos (Associação Brasileira de Cuidados Paliativos, 2013; Bullechek, et al., 2016).

As terapias renais substitutivas - diálise peritoneal, hemodiálise e transplante renal - são indicadas para a manutenção da vida diante da cronicidade da doença renal. Diante da doença renal crônica (DRC) em estágio terminal, pacientes experienciam sintomas físicos, emocionais, psicológicos e espirituais, que impactam na qualidade de vida. Essa última pode ser modificada conforme o entendimento do paciente sobre o processo de adoecimento, a cultura e as vivências com os sintomas e os tratamentos. Dessa forma, faz-se necessária a readequação das intervenções, com base na avaliação individual por meio de instrumentos padronizados e validados (Ferreira \& Pereira, 2020; Tavares et al., 2021).

Nessa fase, indica-se: o uso de hemodiálise incremental, ou seja, aquela utilizada em acordo com a função renal para controlar sintomas; diálise centrada no paciente e paliativa, que têm como finalidade a redução de efeitos do tratamento 
nefrológico; o uso de tratamento conservador abrangente, que consiste em intervenções para reduzir a progressão da doença renal e seus efeitos, englobando o suporte físico, social, emocional, espiritual e familiar; além do planejamento das decisões acerca dos cuidados em conjunto com o paciente, considerando o prognóstico e o tempo de vida estimado, registrando os desejos e as condutas, de forma a promover a qualidade de vida, o conforto e a dignidade diante da DRC em estágio terminal (Tavares et al., 2021).

O Quadro 3 demonstra o registro de visualizações dos vídeos nas plataformas e ainda, no Instagram, foi possível resgatar dados referentes às impressões e às descobertas.

Quadro 3 - Visualizações, impressões e descobertas dos vídeos no YouTube e no Instagram.

\begin{tabular}{|c|c|c|c|c|c|c|}
\hline \multirow{2}{*}{ Plataforma } & \multicolumn{2}{|l|}{ YouTube } & \multicolumn{4}{|l|}{ Instagram } \\
\hline & \multicolumn{2}{|l|}{ Visualizações } & \multicolumn{2}{|l|}{ Visualizações } & \multirow{2}{*}{\begin{tabular}{|l|} 
Impressões \\
$(07 / 2020 \quad$ a \\
$02 / 2021)$
\end{tabular}} & \multirow{2}{*}{\begin{tabular}{|l} 
Descobertas \\
$(07 / 2020-02 / 2021)$
\end{tabular}} \\
\hline Material & $\begin{array}{ll}\text { Mês } & 1 \\
(07 / 2020) & \end{array}$ & Mês 2 (08/2020) & $\begin{array}{ll}\text { Mês } & 1 \\
(07 / 2020) & \end{array}$ & $\left|\begin{array}{ll}\text { Mês } & 2 \\
(08 / 2020) & \end{array}\right|$ & & \\
\hline V1 & 120 & 125 & 292 & 295 & 273 & 196 \\
\hline $\mathbf{V} 2$ & 108 & 111 & 429 & 432 & 209 & 148 \\
\hline V3 & 114 & 121 & 259 & 264 & 225 & 166 \\
\hline V4 & 63 & 69 & 237 & 253 & 255 & 184 \\
\hline V5 & 49 & 58 & 222 & 233 & 172 & 134 \\
\hline V6 & 53 & 56 & 468 & 476 & 268 & 188 \\
\hline V7 & 20 & 28 & 241 & 248 & 224 & 164 \\
\hline V8 & 27 & 29 & 215 & 219 & 205 & 152 \\
\hline V9 & 10 & 13 & 463 & 465 & 252 & 195 \\
\hline V10 & 14 & 20 & 435 & 437 & 260 & 184 \\
\hline V11 & 12 & 12 & 251 & 257 & 193 & 142 \\
\hline V12 & 29 & 53 & 258 & 261 & 221 & 168 \\
\hline V13 & 25 & 74 & 231 & 235 & 217 & 171 \\
\hline V14 & 14 & 17 & 177 & 178 & 202 & 173 \\
\hline V15 & 107 & 117 & 205 & 206 & 196 & 162 \\
\hline V16 & 25 & 25 & 157 & 157 & 174 & 146 \\
\hline V17 & 22 & 26 & 240 & 244 & 216 & 175 \\
\hline V18 & 19 & 20 & 207 & 209 & 235 & 200 \\
\hline V19 & 26 & 29 & 114 & 117 & 155 & 119 \\
\hline V20 & 19 & 24 & 130 & 131 & 160 & 126 \\
\hline V21 & 23 & 23 & 170 & 171 & 223 & 173 \\
\hline
\end{tabular}




\begin{tabular}{|l|l|l|l|l|l|l|}
\hline V22 & 22 & 24 & 145 & 145 & 186 & 142 \\
\hline V23 & 20 & 27 & 149 & 151 & 172 & 144 \\
\hline V24 & 31 & 32 & 197 & 199 & 194 & 163 \\
\hline
\end{tabular}

Fonte: Dados da pesquisa (2021).

Os dados demonstram que à medida que o tempo passa, o número de visualizações dos materiais não aumenta significativamente. Tendo sido observado aumento nos dois primeiros dias após a postagem. Um outro ponto a ser destacado é que, conforme recomendações internacionais, os vídeos com enfoque educativo devem ter, no máximo, entre cinco e seis minutos de duração para que sejam assistidos até o fim (Liu et al., 2019; Oller-Arlandis \& Oller-Arlandis, 2017). Entretanto, os vídeos que tiveram um maior número de visualizações no canal do YouTube (V3, V4 e V12), tiveram um maior tempo de duração comparado ao indicado, com uma média de 10 minutos e 12 segundos.

Os vídeos postados possuem um contador de visitas que informa quantas visualizações uma publicação possui, sendo que, esses dados podem ser divulgados para qualquer pessoa que deseja acompanhá-los. Nessa perspectiva, um vídeo torna-se viral quando muito divulgado em consonância ao tipo de conteúdo (Oller-Arlandis \& Oller-Arlandis, 2017).

Assim, essa contagem feita pela plataforma indica que a influência do vídeo está diretamente relacionada à visualização, ou seja, quanto mais visualizado for, mais influente o vídeo será. Dito isso, é possível perceber que os usuários normalmente consultam a internet como referência para assuntos relacionados à saúde e que a mesma serve como fonte de informação tanto para leigos quanto para profissionais (Mitchell et al., 2017).

Este relato apresenta como limitações o curto tempo de análise do impacto das tecnologias. Destaca-se que a divulgação de cada material pode ter impactado em maior visualização de alguns em detrimento de outros. Houve, ainda, maior alcance regional, já que os seguidores dos perfis eram majoritariamente do município em que o projeto de extensão desenvolvia as ações. Além disso, alguns dados fornecidos pelas plataformas não necessariamente convergem com a realidade, tendo em vista que não é possível verificar a autenticidade junto a cada usuário. Uma última limitação foi o tempo longo dos vídeos, o que pode ter tornado-os menos atrativos para alguns usuários.

\section{Considerações Finais}

Vídeos e folhetos educativos sobre cuidados paliativos demonstraram ser tecnologias educacionais atrativas com potencial de serem consumidas pelos usuários do Instagram e do YouTube, tendo em vista que os resultados obtidos no presente estudo demonstraram um significativo alcance dos materiais postados nas mídias sociais, representado pelo número de descobertas, impressões e visualizações, além do número de usuários que acompanham os perfis do projeto. Abordar assuntos relacionados à terminalidade da vida ainda é considerado um tabu. Entretanto, as mídias sociais podem auxiliar na disseminação de novas formas de cuidar das pessoas que vivenciam uma doença que não responde ao tratamento modificador, o processo de morrer e a morte, e de suas famílias. Sugere-se a validação dos vídeos e folhetos para uso junto a pacientes e familiares, na prática do cuidado em instituições e, na medida do possível, na formação de estudantes e profissionais de saúde.

Em se tratando da implementação desses materiais nos serviços de saúde, se faz importante viabilizar a disponibilidade às pessoas que tenham condições de acesso às mídias sociais e impressão desses para aquelas que não detenham dessas tecnologias, a fim de que esses produtos sejam consumidos e implementados no cuidado e na assistência aos pacientes que experienciam uma doença que ameaça a vida ou que estejam diante da terminalidade.

Mídias sociais, como o Instagram e o YouTube, são um novo ambiente a ser explorado pela enfermagem e pela área da saúde para o desenvolvimento de ações educativas. Temas ainda pouco difundidos e explorados podem ter maior 
aceitabilidade e visibilidade se abordados por tecnologias educacionais que considerem a linguagem e recursos que chamam mais atenção da população. Por fim, sugere-se que futuros estudos, do tipo pesquisa ação ou estudos metodológicos, sejam desenvolvidos junto aos pacientes, profissionais e estudantes de saúde, de maneira a desenvolver e validar materiais educativos, por meio de tecnologias educacionais, verificando sua aplicabilidade nas instituições de saúde e os impactos na assistência.

\section{Referências}

Associação Brasileira de Cuidados Paliativos. (2013). Consenso Brasileiro de náuseas e vômitos em cuidados paliativos. Revista Brasileira de Cuidados Paliativos. https://nutritotal.com.br/pro/wp-content/uploads/sites/3/2011/08/222-ConsensoNauseaVomito.pdf

Bullechek, G. M., Butcher, H. K., Dochterman, J. M., \& Wagner, C. M. (2016). Classificação das intervenções de enfermagem (6o ed). Elsevier.

Centers for Disease Control and Prevention. (2012). Social media guidelines and best practices: CDC YouTube channel. Department of Health and Human Services. https://www.cdc.gov/SocialMedia/Tools/guidelines/pdf/onlinevideo.pdf

Costa, S. V. da, \& Ceolim, M. F. (2013). Fatores que interferem na qualidade do sono de pacientes internados. Revista da Escola de Enfermagem da USP, 47, 46-52. https://doi.org/10.1590/S0080-62342013000100006

Dadalto, L. (2016). A necessidade de um modelo de Diretivas Antecipadas de Vontade para o Brasil: Estudo comparativo dos modelos português e franceses. Revista M. Estudos sobre a morte, os mortos e o morrer, 1(2), 443-460. https://doi.org/10.9789/2525-3050.2016.v1i2.443-460

Dadalto, L., Tupinambás, U., \& Greco, D. B. (2013). Diretivas antecipadas de vontade: Um modelo brasileiro. Revista Bioética, 21 , $463-476$.

Ferreira, M. M. de M., \& Pereira, L. T. C. (2020). Qualidade de vida de pacientes renais crônicos terminais em uso de terapia renal substitutiva. Revista Enfermagem Contemporânea, 9(2), 265-278. https://doi.org/10.17267/2317-3378rec.v9i2.2962

IASP. (2010). Guide to pain management In Low-Resource Settings. iasp/files/production/public/Content/ContentFoldersPublications2/FreeBooks/GuidetoPainManagement_Portuguese.pdf

IASP Terminology-IASP. ([s.d.]). Recuperado 24 de maio de 2021, de https://www.iasp-pain.org/Education/Content.aspx?ItemNumber=1698 Instagram do Facebook. (2021). About us [Blog]. Instagram. https://about.instagram.com/blog

Lee, E., Lee, J.-A., Moon, J. H., \& Sung, Y. (2015). Pictures Speak Louder than Words: Motivations for Using Instagram. Cyberpsychology, Behavior, and Social Networking, 18(9), 552-556. https://doi.org/10.1089/cyber.2015.0157

Lewis, M. J. M., Kohtz, C., Emmerling, S., Fisher, M., \& Mcgarvey, J. (2018). Pain control and nonpharmacologic interventions. Nursing2021, 48(9), 65-68. https://doi.org/10.1097/01.NURSE.0000544231.59222.ab

Li, F., Larimo, J., \& Leonidou, L. C. (2021). Social media marketing strategy: Definition, conceptualization, taxonomy, validation, and future agenda. Journal of the Academy of Marketing Science, 49(1), 51-70. https://doi.org/10.1007/s11747-020-00733-3

Lima, T. J. A. de, Lima, M. V. C. de, Oliveira, K. K. D. de, \& Ferreira, V. O. (2020). A Imagem do Enfermeiro no Instagram no Contexto da Pandemia da Covid-19. Enfermagem em Foco, 11(1 Esp), Article 1 Esp. https://doi.org/10.21675/2357-707X.2020.v11.n1 Esp.3702

Liu, M., Cardenas, V., Zhu, Y., \& Enguidanos, S. (2019). YouTube Videos as a Source of Palliative Care Education: A Review. Journal of Palliative Medicine, 22(12), 1568-1573. https://doi.org/10.1089/jpm.2019.0047

Maniva, S. J. C. de F., Carvalho, Z. M. de F., Gomes, R. K. G., Carvalho, R. E. F. L. de, Ximenes, L. B., Freitas, C. H. A. de, Maniva, S. J. C. de F., Carvalho, Z. M. de F., Gomes, R. K. G., Carvalho, R. E. F. L. de, Ximenes, L. B., \& Freitas, C. H. A. de. (2018). Tecnologias educativas para educação em saúde no acidente vascular cerebral: Revisão integrativa. Revista Brasileira de Enfermagem, 71, 1724-1731. https://doi.org/10.1590/0034-7167-2017-0041

Mitchell, I. A., Schuster, A. L. R., Lynch, T., Smith, K. C., Bridges, J. F. P., \& Aslakson, R. A. (2017). Why don't end-of-life conversations go viral? A review of videos on YouTube. BMJ Supportive \& Palliative Care, 7(2), 197-204. https://doi.org/10.1136/bmjspcare-2014-000805

Monteiro, D. da R., Almeida, M. de A., \& Kruse, M. H. L. (2013). Tradução e adaptação transcultural do instrumento Edmonton Symptom Assessment System para uso em cuidados paliativos. Revista Gaúcha de Enfermagem, 34(2), 163-171.

Most used social media 2020. (2021). Statista. https://www.statista.com/statistics/272014/global-social-networks-ranked-by-number-of-users/

Nietsche, E. A., Backes, V. M. S., Colomé, C. L. M., Ceratti, R. do N., \& Ferraz, F. (2005). Tecnologias educacionais, assistenciais e gerenciais: Uma reflexão a partir da concepção dos docentes de enfermagem. Revista Latino-Americana de Enfermagem, 13(3), 344-352. https://doi.org/10.1590/S010411692005000300009

Oliveira, D. S. da Si., Roque, V. de A., \& Maia, L. F. dos S. (2019). A dor do paciente oncológico: As prinncipais escalas de mensuração. 9(26), 40-59.

Oller-Arlandis, V., \& Oller-Arlandis, V. E. (2017). YouTube como fuente de información sanitaria y divulgación del conocimiento sobre Cuidados Paliativos en el domicilio. Hospital a Domicilio, 1(3), 153-166-166. https://doi.org/10.22585/hospdomic.v1i3.23

Palliaguide.be_Traitement de la nausée en soins palliatifs. ([s.d.]). Palliaguide.be - Guidelines en Soins Palliatifs. Recuperado 24 de maio de 2021, de http://www.palliaguide.be/nausees-vomissements-soins-palliatifs/ 
Research, Society and Development, v. 10, n. 7, e22310716534, 2021

(CC BY 4.0) | ISSN 2525-3409 | DOI: http://dx.doi.org/10.33448/rsd-v10i7.16534

Santos, E. C. dos, Oliveira, I. C. M. de, \& Feijão, A. R. (2016). Validação de protocolo assistencial de enfermagem para pacientes em cuidados paliativos. Acta Paulista de Enfermagem, 29, 363-373. https://doi.org/10.1590/1982-0194201600051

Sobre o YouTube-YouTube. ([s.d.]). https://www.youtube.com/about/

Souza, T. dos S. de, Ferreira, F. B., Bronze, K. M., Garcia, R. V., Rezende, D. F. de, Santos, P. R. dos, \& Gadelha, S. R. (2020). Mídias sociais e educação em saúde: O combate às Fake News na pandemia da COVID-19. Enfermagem em Foco, 11(1.ESP), Article 1.ESP. https://doi.org/10.21675/2357707X.2020.v11.n1.ESP.3579

Statista. (2021). Social media usage in Brazil - statistics \& facts. Statista. https://www.statista.com/topics/6949/social-media-usage-in-brazil/

Tavares, A. P. dos S., Santos, C. G. da S., Tzanno-Martins, C., Barros Neto, J., Silva, A. M. M. da, Lotaif, L., Souza, J. V. L., Tavares, A. P. dos S., Santos, C. G. da S., Tzanno-Martins, C., Barros Neto, J., Silva, A. M. M. da, Lotaif, L., \& Souza, J. V. L. (2021). Cuidados de suporte renal: Uma atualização da situação atual dos cuidados paliativos em pacientes com DRC. Brazilian Journal of Nephrology, 43(1), 74-87. https://doi.org/10.1590/2175-8239-jbn-2020-0017

Westphal Hahn, F., \& Cordeiro, F. R. (2021). Estratégias para o desenvolvimento de educação em saúde sobre dor no hospital. Research, Society and Development, 10(3), e25210313297. https://doi.org/10.33448/rsd-v10i3.13297 